\title{
Quantitative Evaluation of Low-Cost Frame-Grabber Boards for Personal Computers
}

\author{
James M. Kofler, Jr, Joel E. Gray, James T. Fuelberth, and Jerome P. Taubel
}

\begin{abstract}
Nine moderately priced frame-grabber boards for both Macintosh (Apple Computers, Cupertino, CA) and IBMcompatible computers were evaluated using a Society of Motion Pictures and Television Engineers (SMPTE) pattern and a video signal generator for dynamic range, gray-scale reproducibility, and spatial integrity of the captured image. The degradation of the video information ranged from minor to severe. Some boards are of reasonable quality for applications in diagnostic imaging and education. However, price and quality are not necessarily directly related.

Copyright $(\mathcal{C} 1995$ by W.B. Saunders Company
\end{abstract}

KEY WORDS: quality control, video, frame grabber, personal computers, analog devices, picture archiving and communication systems (PACS).

$\mathbf{T}$ HE CURRENT popularity of multimedia has resulted in an increase in the amount of commercially available multimedia hardware and software. Competition for the multimedia market has produced a number of low-cost ( $\$ 400$ to $\$ 4,000$ ) frame-grabber boards for both the Macintosh (Apple Computers, Cupertino, $\mathrm{CA})$ and personal computer (PC) platforms. The combination of low-cost frame-grabber boards, powerful desktop computers, and sophisticated image-analysis software fosters the concept of using computer-aided image evaluation in diagnostic medical imaging.

In computer-aided image analysis of radiographic images, the analog film (or video image) is quantized into discrete pixel values. The pixel values can then be measured or altered in essentially any manner desired. The seemingly unbounded potential of computer-aided image analysis distracts from the fact that the digitized image is a reproduction of the analog image and does not necessarily contain the same amount or quality of information. Image digitization by means of digitizing the video signal typically does not preserve the entire information content of the film. Furthermore, the framegrabbing process can introduce artifacts into the digital image. Measurements or analysis of the resulting digital image can be severely altered by the imperfections of the frame-grabbing process.

When analyzing medical images, strict attention must be paid to every component in the imaging chain that can alter the image information. In conventional diagnostic radiologic quality control, an effort is made to assure that image degradation caused by any component of the imaging chain is understood and minimized. ${ }^{1,2}$ Extrapolation of this concept to computer-aided image analysis dictates that the additional items in the imaging chain, namely the video camera, digitizing process, and display must also be subject to such scrutiny.

\section{MATERIALS AND METHODS}

Five Macintosh and four PC frame-grabber boards (Table 1) were evaluated using a video signal generator (Signal Source 202; VII Visual Information Institute, Inc, Dayton, $\mathrm{OH}$ ) and a Society of Motion Pictures and Television Engineers (SMPTE) pattern generator (TPG 640 Test Pattern Generator; Bildsystem, Malmö, Sweden). The test patterns that were used provided industry-standard video signals ${ }^{3}$ to investigate specific characteristics of the digitization process as suggested by Rowberg. ${ }^{4}$ With the exception of one frame-grabber board $(\mathrm{H})$, all of the boards in this study were either dedicated for gray scale or set by the software to capture in gray scale mode. Images captured with board $\mathrm{H}$, which was a color board, were converted to gray scale using Photoshop (version 2.0; Adobe Systems, Mountain View, CA) before analysis.

The video test patterns that were used in this study are listed in Table 2 along with the method of analysis used for each pattern. Analysis of digital images was performed visually and quantitatively using the public domain National Institutes of Health (NIH) Image software package (version 1.49 , written by Wayne Rasband at the NIH and available over the Internet by anonymous file transfer protocol from zippy.nimh.gov or on floppy disk from National Technical Information Service (NTIS), 5285 Port Royal Rd, Springfield, VA 22161; part number PB93-504868) on a Macintosh IIci PC.

Although some of the boards offered options to average multiple frames, image acquisition consisted of grabbing a single frame of gray scale video signal to allow for comparison among all boards. Any other software enhancements, such as smoothing, were disabled. The boards were then rated for each performance test according to the following

From the Department of Diagnostic Radiology and Division of Information Services, Mayo Clinic and Foundation, Rochester, $M N$.

Address reprint requests to James M. Kofler, Jr, Department of Diagnostic Radiology, Mayo Clinic and Foundation, 200 First St SW, Rochester, MN 55905.

Copyright $\odot 1995$ by W.B. Saunders Company 0897-1889/95/0804-0005\$3.00/0 
Table 1. Frame-Grabber Boards Used in Study

\begin{tabular}{|c|c|c|c|}
\hline Reference & Manufacturer* & Model & Suggested Retail Pricet \\
\hline \multicolumn{4}{|l|}{ Macintosh } \\
\hline$A$ & Digital Vision, Inc & Computer Eyes-RT for Mac & $\$ 600$ \\
\hline B & Data Translation, Inc & QuickCapture for Mac (DT2255 rev. 1.1) & $\$ 1,295$ \\
\hline $\mathrm{C}$ & Data Translation, Inc & QuickCapture for Mac (DT2255 rev. 1.23) & $\$ 1,295$ \\
\hline $\mathrm{D}$ & RastorOps & $24 \times L T V$ & $\$ 3,499$ \\
\hline$E$ & Scion Corp & LG-3 Scientific Frame Grabber & $\$ 895$ \\
\hline \multicolumn{4}{|c|}{ IBM-Compatable } \\
\hline $\mathrm{F}$ & Digital Vision, Inc & Computer Eyes-RT Mono for PC & $\$ 300$ \\
\hline G & Matrox Electronic Systems & Image One & $\$ 2,995$ \\
\hline $\mathrm{H}$ & Creative Labs & Video Spigot for Windows & $\$ 500$ \\
\hline I & Data Translation, Inc & DT3852 for PC & $\$ 3,995$ \\
\hline
\end{tabular}

*Manufacturer locations are as follows: Digital Vision, Inc (Dedham, MA); Data Translation, Inc (Marlboro, MA); RastorOps (Santa Clara, CA); Scion Corp (Frederick, MD); Matrox Electronic Systems (Dorval, Quebec, Canada); Creative Labs, Inc (Milpitas, CA).

†Suggested retail prices are from September 1993.

relative scale: poor, below average, average, above average, or excellent. The board was rated as poor if the image was severely degraded from the input video signal. Possible causes could be excessive digitizing artifacts, noise, or distortion. A poor image was essentially useless for performing quantitative studies for the specified test. The board was rated as below average if image degradation was severe but the digital image somewhat represented the input video signal for the specified test. The board was rated as average if image degradation was present, but not excessive. The board was rated as above average if the digital image showed only minor degradation. The board was rated as excellent if the digital image entirely preserved the image information of the video signal for the specified test.

A number of rating criteria for each test pattern were used.

Dynamic range and linearity. The gray-scale step images were judged based on the range of pixel values present and on the relative sizes of each step.

Resolution. The number of complete resolution patterns on the column-averaged plots as well as the modulation of each pattern was noted to grade the image.

Edge response. The response of the board when presented with a $100 \%$-to-black-level interface was evaluated

Table 2. Description of Video Patterns Used in Study

\begin{tabular}{|c|c|c|}
\hline Video Test Pattern & Pattern Description & Method of Digital Image Analysis \\
\hline Burst & $\begin{array}{l}\text { A series of vertical resolution patterns (1.28 MHz, } 1.92 \\
\mathrm{MHz}, 2.56 \mathrm{MHz}, 3.21 \mathrm{MHz}, 4.20 \mathrm{MHz} \text { ) with } 100 \% \\
\text { video signal modulation. Used to measure resolu- } \\
\text { tion. }\end{array}$ & $\begin{array}{l}\text { To obtain a column average, each column of pixels } \\
\text { was averaged over the entire height of the image. } \\
\text { The averages were plotted versus pixel position } \\
\text { across the image. }\end{array}$ \\
\hline Gray scale & $\begin{array}{l}\text { Video signal steps (vertical bars) that range from } 0 \% \text { to } \\
100 \% \text { video signal. Used to measure dynamic range } \\
\text { and linearity. }\end{array}$ & $\begin{array}{l}\text { To obtain a column average, each column of pixels } \\
\text { was averaged over the entire height of the image. } \\
\text { The averages were plotted versus pixel position } \\
\text { across the image. }\end{array}$ \\
\hline Window & $\begin{array}{l}\text { A } 100 \% \text { video signal square patch surrounded by a } \\
0 \% \text { signal background. Used to measure edge } \\
\text { response. }\end{array}$ & $\begin{array}{l}\text { The columns of pixels that contained the window were } \\
\text { averaged and plotted. This method was repeated in } \\
\text { the horizontal direction using the rows of pixels. }\end{array}$ \\
\hline Mesh & $\begin{array}{l}\text { A } 100 \% \text { video signal mesh pattern (at least } 1 \mathrm{~cm} \times 1 \\
\mathrm{~cm} \text { ) on a } 0 \% \text { signal background. Used to measure } \\
\text { spatial distortion. }\end{array}$ & $\begin{array}{l}\text { A column average of five pixels was obtained at the } \\
\text { left, center, and right locations of the image. The dis- } \\
\text { tances to the center of each mesh line were calcu- } \\
\text { lated. The same method was applied to the hori- } \\
\text { zontal direction using rows of pixels at the top, } \\
\text { center and bottom of the image. }\end{array}$ \\
\hline Flat field & $\begin{array}{l}\text { A } 50 \% \text { video signal over the entire display area. Used } \\
\text { to measure the noise value, structure artifacts, and } \\
\text { pixel gradients (shading). }\end{array}$ & $\begin{array}{l}\text { The mean and standard deviation of the pixel values } \\
\text { were calculated over the entire image. Also used to } \\
\text { determine structured noise and any pixel value } \\
\text { trends throughout the image. }\end{array}$ \\
\hline SMPTE & $\begin{array}{l}\text { Gray-scale steps from } 0 \% \text { to } 100 \% \text { video signal, } 5 \% \\
\text { (light and dark) contrast patches, and resolution pat- } \\
\text { terns at all corners and in the center of the image. } \\
\text { Used for low-contrast visualization and overalt } \\
\text { image quality. }\end{array}$ & $\begin{array}{l}\text { Visual inspection of the low-contrast patches, text, } \\
\text { gray-scale steps, and resolution patterns. }\end{array}$ \\
\hline
\end{tabular}


Fig 1. Summary of the framegrabber board rating.

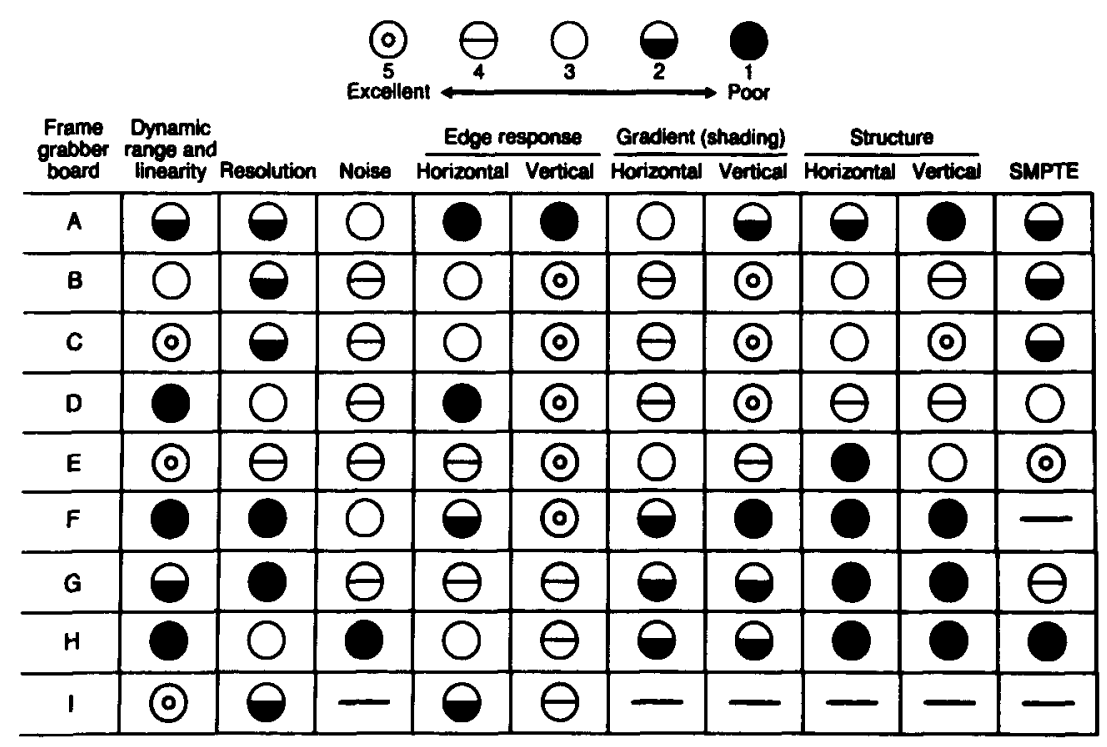

using column- and row-averaged plots of the window test pattern. An effort was made to judge the image only based on the response at the interface and not on the baseline pixel value or noise.

Spatial linearity. The deviation of the distances measured between the grid pattern lines were used to calculate the spatial distortion.

Noise. The standard deviation of the entire flat-field image ( $50 \%$ video) was used as a measure of the noise. Note that signal-to-noise ratio was not used because the boards produced different mean pixels values even though the input video signal was identical for all boards.

Pixel gradient. Column and row averages of the flat-field images were plotted and analyzed for any increasing or decreasing pixel value trends across the image.

Structure artifacts. The same plots used for the pixel gradient study were used to evaluate any structure that was present in the image. Because the plots represent column or row averages, any deviations from the mean value were considered to be structural noise.

Overall preservation of image integrity. The SMPTE pattern was used to visually inspect the images for overall appearance. Although the SMPTE pattern examines many of the same characteristics evident in the other test patterns, the SMPTE pattern provides a familiar image with a variety of relevant information. The SMPTE images were judged on the presence, or absence, of the low-contrast patches, the appearance of alphanumeric characters, the horizontal and vertical lines, the gray scale steps, and the resolution patterns.

\section{RESULTS}

The results show that all of the frame-grabber boards investigated degrade the image quality to various degrees and alter the spatial information of the video input signal.

The graded analysis of each of the framegrabber boards is shown in Fig 1. A comparison of price versus performance is shown in Fig 2. The overall results can be summarized as follows:

Dynamic range and linearity. The dynamic ranges of the frame-grabber boards were rated from poor to excellent (Fig 3). Boards that showed exceptional dynamic range and linearity were $\mathrm{C}, \mathrm{E}$, and $\mathrm{I}$. Boards with inferior dynamic range exhibited one or more of the following: a high baseline, complete loss of gray-scale steps, and severe overshoots or undershoots at the step interface, which interfered with the grayscale information.

Resolution. None of the studied boards completely retained all of the burst-pattern information (Figs 4 and 5A). However, one board (E) preserved most of the resolution patterns with $100 \%$ modulation (Fig 5B). Most of the boards, but not all, were able to correctly digitize at

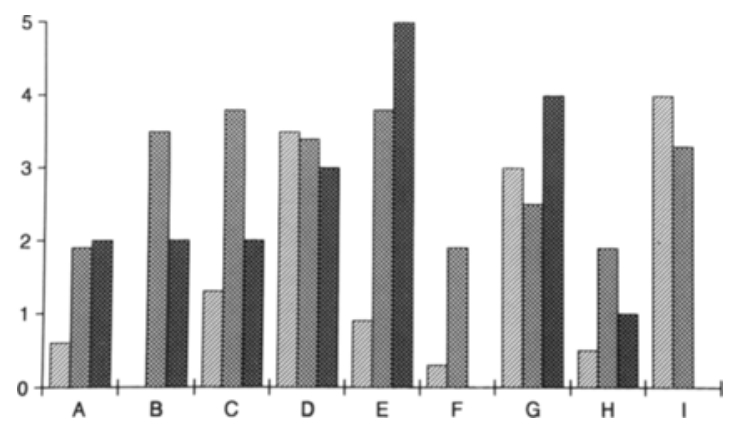

Fig 2. The overall score (average of all scores) (啉), the SMPTE pattern score ( $\square$ ), and suggested retail price for each frame-grabber board evaluated (in thousands of dollars) (圈). 

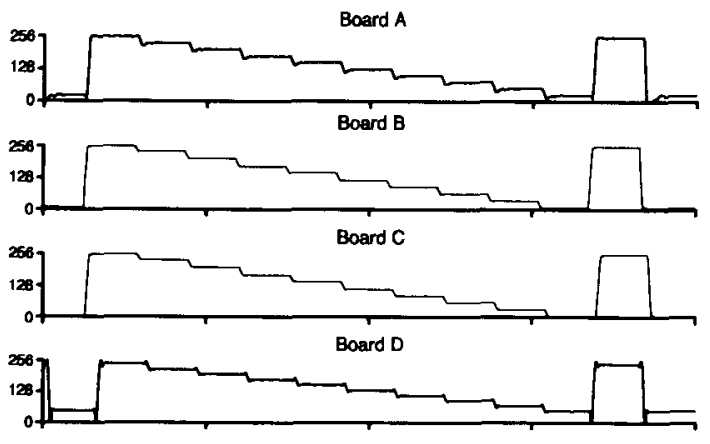

Board E
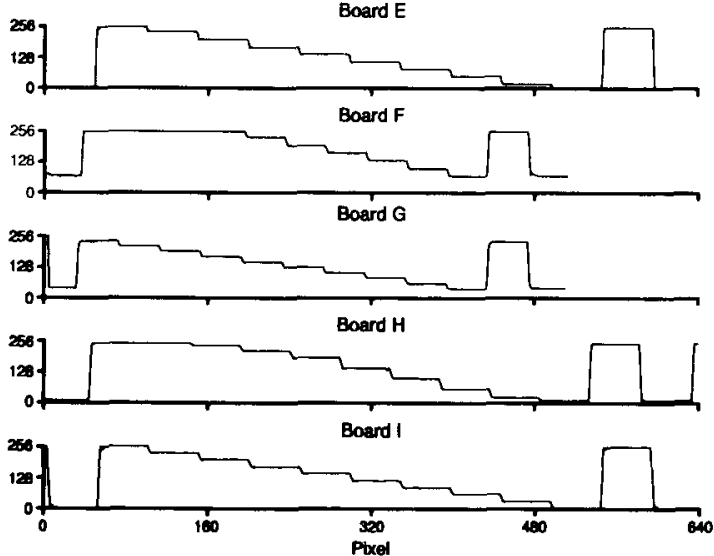

Fig 3. The column-averaged plots of the gray-scale step images for each frame-grabber board.
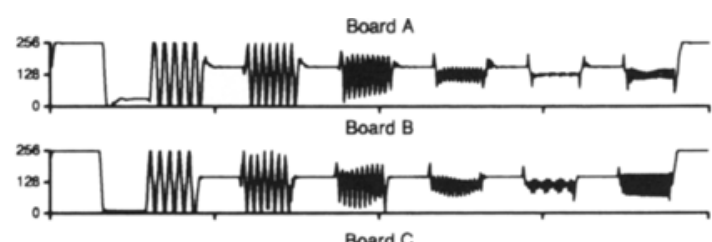

Board C
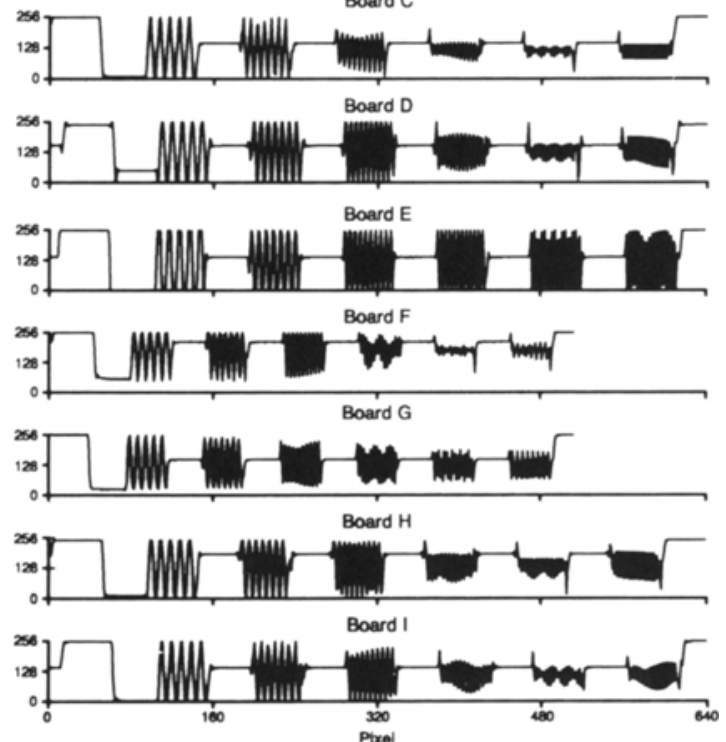

Fig 4. The column-averaged plots of the resolution pattern (burst) images for each frame-grabber board. least the first two resolution patterns (1.28 and $1.92 \mathrm{MHz}$ ) with the modulation being dictated by the dynamic range.

Edge response. Only one board $(\mathrm{E})$ showed an exceptional edge response in both the horizontal and vertical directions (Fig 6A). All of the other boards showed moderate to severe overshooting or undershooting at the interface of the black-level and $100 \%$ video signal (Fig 6B).

Spatial linearity. Only one board showed any significant spatial distortion. Board $\mathrm{E}$ exhibited a shift of 2 to 3 pixels in the horizontal direction that was predominant in the upper half of the digitized image.

Noise. Most of the frame-grabber boards showed a standard deviation of pixel values (over the entire flat-field image) ranging from 0.33 to 0.82 . Three of the boards (A, F, and $\mathrm{H})$ rated poorly with standard deviations of 2.39 ,
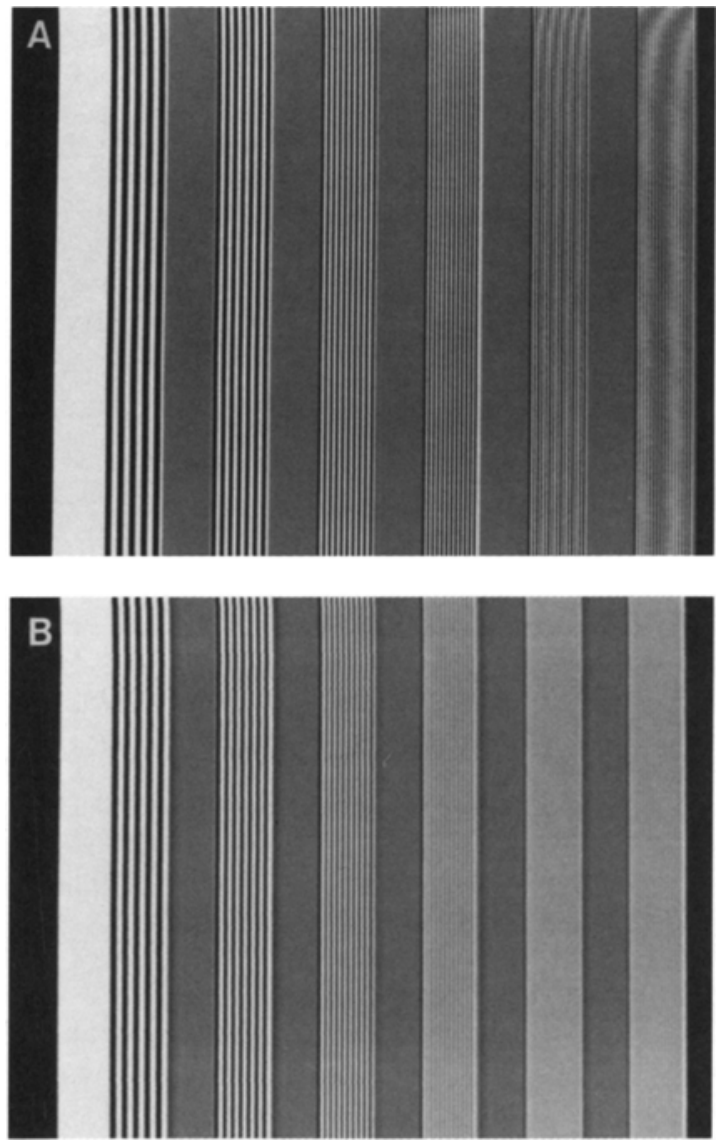

Fig 5. Photographs of the monitor showing (A) above average reproduction of the resolution test pattern (board $E$ ) and $(B)$ poor reproduction of the resolution pattern image (board A). 


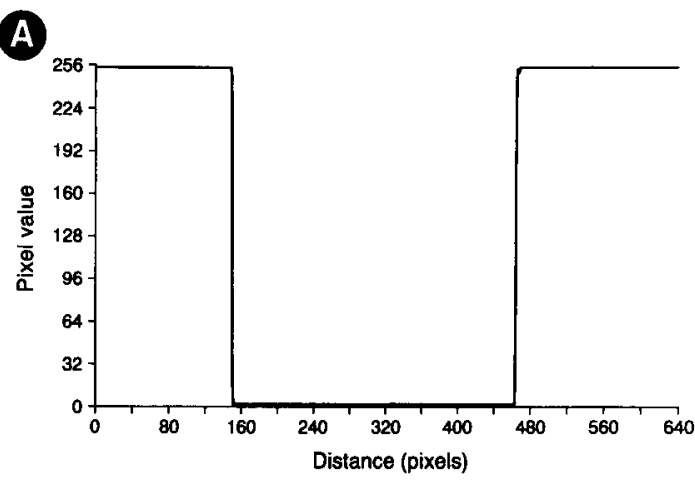

B

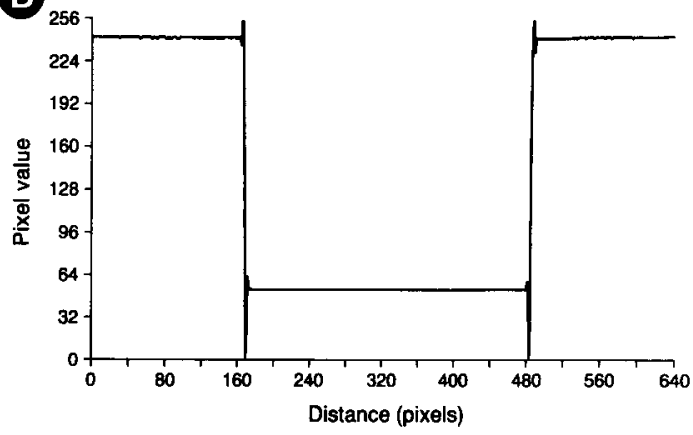

Fig 6. The column-averaged plots showing (A) exceptional edge response (board $E$ ) and (B) poor edge response (board $D$ ).

2.19 , and 14.6 , respectively. Recall that board $\mathrm{H}$ was the only color board evaluated in this study. It is interesting to note that none of the boards provided a mean pixel value of 127 , which is the value expected from the $50 \%$ video signal. It should be emphasized that only a single video frame was used to acquire the images.

Pixel gradient. Most of the boards exhibited an overall flat response ( \pm 1 pixel) across the majority of the flat-field image (in both directions). Boards $\mathrm{A}, \mathrm{F}, \mathrm{G}$, and $\mathrm{H}$ exhibited an anomaly at one end of image in either the horizontal, vertical, or both directions. The anomaly represents a spike or other type of variation that is not consistent with the remainder of the pixel values (see Digitizing structure artifacts, below). The plot of the flat field (vertical direction) for board $\mathrm{F}$ showed a gradient of 5 pixel values from top to bottom (Fig 7). Boards B, C, and D showed an exceptionally flat response across the entire image in the vertical direction; however, board $\mathrm{C}$ exhibited a gradient of 2 pixel values in the horizontal direction.

Structure artifacts. Structure artifacts were evident in nearly all of the images (Fig 8). The predominance of the structure varied among

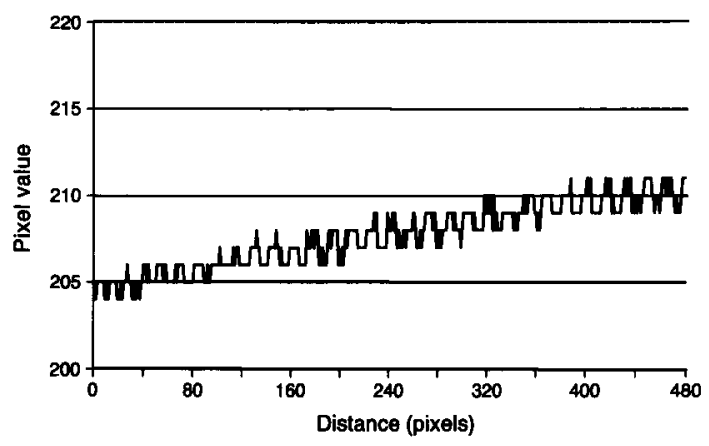

Fig 7. The row-averaged plot of the flat-field image $\mathbf{5 0} \%$ video) showing an increasing trend in the pixel values across the image (from top to bottom). The image was acquired using board $F$.

the boards and is reflected in the standard deviation of the pixel values (see Noise, above). The structure is typically manifested by a series of lines across the image (in either or both directions). Boards that showed the least amount of structure in the horizontal and vertical directions were $\mathrm{B}, \mathrm{C}$, and $\mathrm{D}$.

Overall preservation of image integrity. Visual analysis of the overall preservation of image quality as obtained by a SMPTE pattern showed board $\mathrm{E}$ (Fig 9A) to be superior to the remaining boards. Common problems evident with the SMPTE pattern images include loss of lowcontrast patches and overall image blur (Fig 9B).

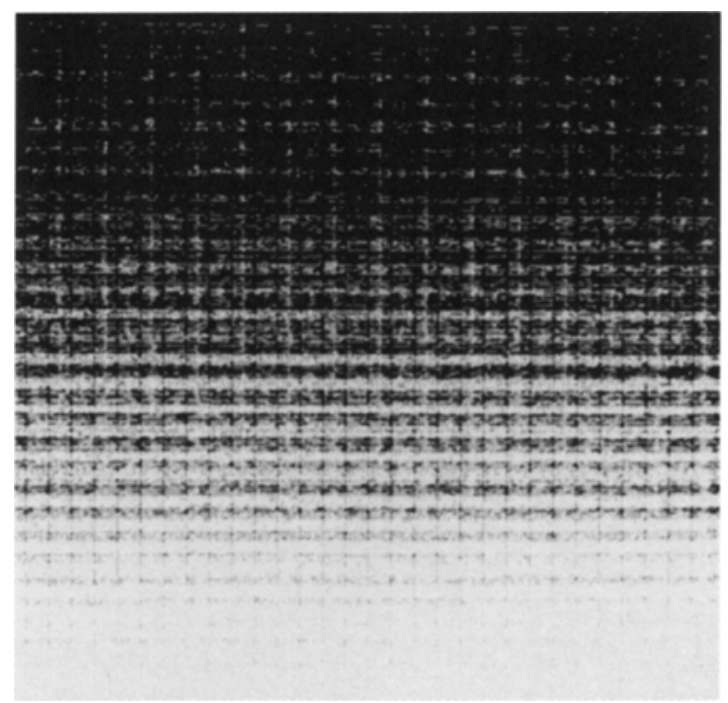

Fig 8. Photograph of the monitor showing the structure that is present in the flat-field image of board $F$. The image is being displayed in black and white (using the threshold feature of Image 1.49 software) to better visualize the structure. 

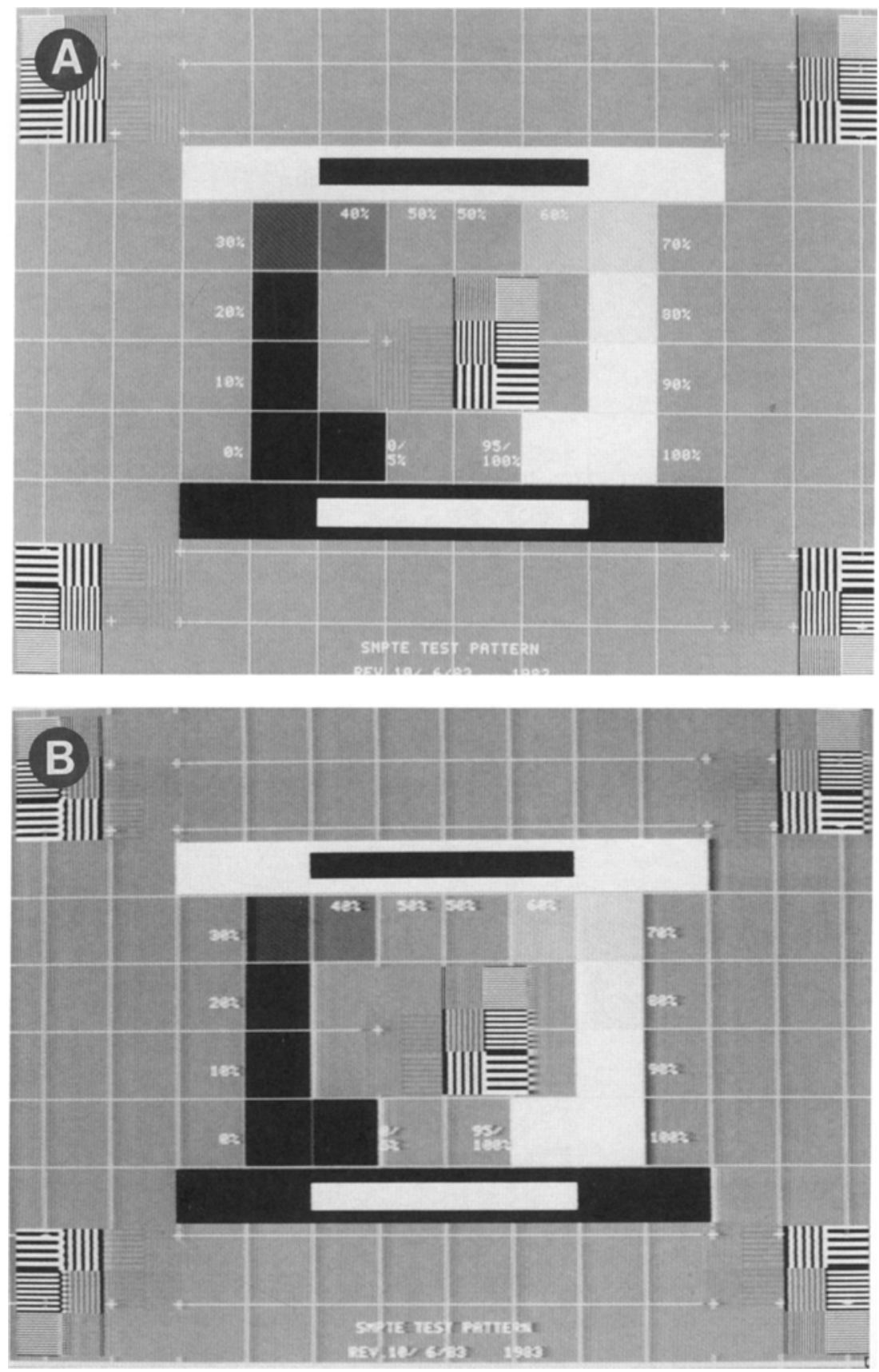

Fig 9. Photographs of the monitor showing (A) exceptional reproduction of the SMPTE test pattern (board E) and (B) poor reproduction of the SMPTE pattern image (board A).

\section{DISCUSSION}

The results show that the quality of the digital image is negatively influenced by the framegrabber board that was used to digitize the image. The magnitude of the image degradation is dependent on the specific value of interest. For example, it was shown that board E introduced an unacceptable amount of structural noise into the image; however, the SMPTE pattern image of board $E$ was far superior to the remaining boards. This implies that if a perfectly uniform field is essential for proper image analysis, then board $E$ would not be the frame grabber of choice. If resolution and overall image quality were the issues, then board $\mathrm{E}$ would be the desired board. Therefore, when deciding which frame-grabber board to purchase, one must consider the application for 
which it will be used and which particular board characteristics will be of importance. Additionally, one should note that the price of the frame-grabber board does not necessarily reflect the performance of the board.

Some additional items of consideration include software and hardware features, software and hardware compatibility, and price. Multiple frame averaging can significantly reduce the noise, but not necessarily the structural artifacts, significantly. Many boards that offer frame averaging also allow for acquiring cine sequences. Applications that do not require color images should use only gray-scale frame-grabber boards. The color board that was evaluated in this study scored reasonably well in the tests for edge response; however, the overall image quality as assessed from the SMPTE pattern was very poor. Compatibly with existing or future equipment should also be considered when purchasing a frame-grabber board. Some questions to consider are the following: Will the board work on the computer in its current configuration? If not, what additional items need to be purchased? Is the software usable for the intended operation? Can images be saved in a format which is needed by any other applications? Is the bit depth acceptable?

All of the frame grabbers evaluated degraded the quality of the image information that was input as a video signal from a signal generator. The results imply that quantitative analysis of images that have been digitized by means of frame grabbing is limited by the quality of the digitizing board. Failure to acknowledge the degradation of the digitizing process can lead to erroneous and misleading results. Therefore, measurements of the frame-grabber characteristics should be performed before purchasing and attempting any quantitative analysis of digital images, and the limitations introduced by the frame-grabber board must be recognized.

\section{REFERENCES}

1. Gray JE, Winkler NT, Stears J, et al: Quality Control in Diagnostic Radiology. Gaitherburg, MD, Aspen, 1983

2. National Council on Radiation Protection Report No. 99. Quality Assurance for Diagnostic Imaging. NCRP, Bethesda, MD, 1988
3. Grob B: Basic Television and Video Systems. New York, NY, McGraw-Hill, 1984, pp 200-211

4. Rowberg AH: Objective analysis of image quality of video capture systems. Presented at SPIE Medical Imaging IV, Conference Proceedings, 1232:61-70, 1990 